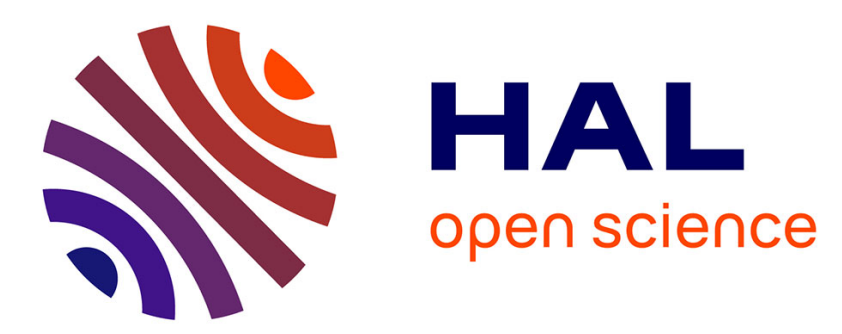

\title{
Evolution of LSPR of gold nanowire chain embedded in dielectric multilayers
}

\author{
Zakariae Oumekloul, S. Lahlali, A. Mir, Abdellatif Akjouj
}

\section{To cite this version:}

Zakariae Oumekloul, S. Lahlali, A. Mir, Abdellatif Akjouj. Evolution of LSPR of gold nanowire chain embedded in dielectric multilayers. Optical Materials, 2018, 86, pp.343-351. 10.1016/j.optmat.2018.10.020 . hal-03185124

\section{HAL Id: hal-03185124 \\ https://hal.science/hal-03185124}

Submitted on 18 Aug 2021

HAL is a multi-disciplinary open access archive for the deposit and dissemination of scientific research documents, whether they are published or not. The documents may come from teaching and research institutions in France or abroad, or from public or private research centers.
L'archive ouverte pluridisciplinaire HAL, est destinée au dépôt et à la diffusion de documents scientifiques de niveau recherche, publiés ou non, émanant des établissements d'enseignement et de recherche français ou étrangers, des laboratoires publics ou privés.

\section{(c)(1)}

Distributed under a Creative Commons Attribution| 4.0 International License 


\title{
Evolution of LSPR of gold nanowire chain embedded in dielectric multilayers
}

\author{
Z. Oumekloul ${ }^{\mathrm{a}, \mathrm{b}, *}$, S. Lahlali ${ }^{\mathrm{a}, \mathrm{b}}$, A. Mir ${ }^{\mathrm{a}}$, A. Akjouj ${ }^{\mathrm{b}}$

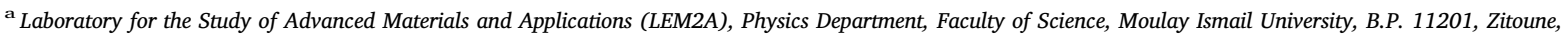 \\ Meknes, Morocco \\ ${ }^{\mathrm{b}}$ Univ. Lille, Department of Physics, UMR 8520 - Institute of Electronics, Microelectronics and Nanotechnologies, F-59650, Lille, France
}

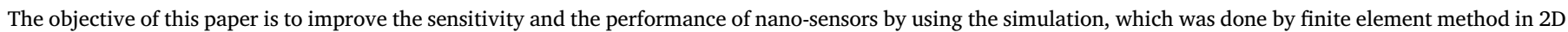

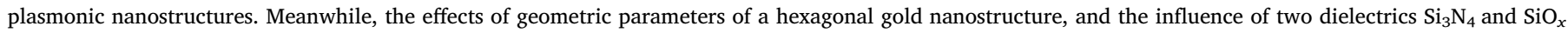

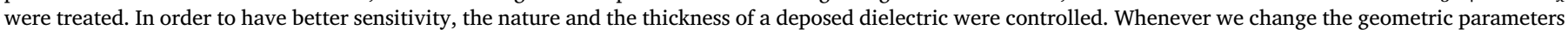

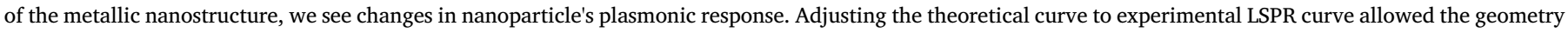

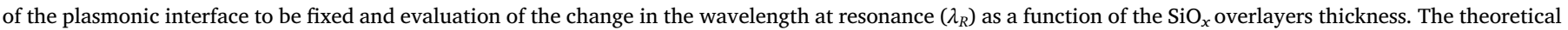
data were compared with experimental results obtained on glass/Au nanowires/SiO ${ }_{x}$ and glass/Au Nanowires/Si ${ }_{3} \mathrm{~N}_{4}$ interfaces.

\section{Introduction}

The terms "nanomaterial" and "nanostructured material" appeared in the early 90s, but the study and use of nanomaterials are older. The colloids are the first community of this functionalized state of matter. However, a colloidal system was rarely perceived as a material, but rather as a fluid with special properties. Michael Faraday already studied the manufacture of nanoparticles by colloidal chemistry in the 19th century in the 1850s [1]. Faraday was well aware that colloidal gold behave very differently from macroscopic grain size vis-a-vis the visible light.

Currently there is strong demand for bio-sensing devices based on surface plasmon resonance (SPR) of noble metal nanoparticles in specific silver and gold deposited on functionalized surfaces [2-17]. Optical properties of metallic nanomaterials are determined by a set of parameters such as the chemical composition of the substrate in which the metal is deposited, the size particles and form, as well as the environment surrounding these metal nanomaterials. The LSPR are very sensitive to modifications in the medium close the surfaces. The effects of the refractive index of the surrounding medium on the plasmonic band LSPR (Localized Surface Plasmon Resonance) have been studied for many years [17-20]. Schultz studied the influence of the refractive index of the surrounding single silver nanoparticles in various oils [21].
After adding oil, the color of silver nanoparticles is changed from blue to green, indicating a shift to red color. The color of the modified nanoparticles turns blue when removing the oil. In the same year, Van Duyne uses silver nanoparticles as probes for detecting the formation of a monolayer on the surface of the nanoparticles [22].

The nanosensors based on LSPR have several advantages for bio sensing since it presents high sensitivity by detecting changes in the refractive index with no label and is flexible for the detection of biomolecular interactions of nanoparticles [19,20,23-26]. On top of that, the detection is in real time and allows the use of micro fluidic systems, low cost and ease for the instrumental configuration. These benefits show that these nanosensors can be applied in a wide range of areas, such as the medical field [20,27-30], that of food security [31,32], or the one on the monitoring of the environment [19].

In this work, we will numerically simulate the optical response of metal nanoparticles by changing the peak LSPR wavelength, for a hexagonal geometry based on the Drude-Lorentz-model for gold deposited on a glass substrate. On one hand, our study is carried out according to different geometric parameters of this hexagonal structure, and on the other hand, it depends on the thickness of the dielectric layers of $\mathrm{SiO}_{x}$ and $\mathrm{Si}_{3} \mathrm{~N}_{4}$ deposited above the gold nanoparticles. Calculations are performed using the finite element method (FEM), which solves Maxwell's equations. The aim of our work is not only to

\footnotetext{
${ }^{*}$ Corresponding author. Laboratory for the Study of Advanced Materials and Applications (LEM2A), Physics Department, Faculty of Science, Moulay Ismail University, B.P. 11201, Zitoune, Meknes, Morocco.

E-mail address: z.oumekloul@edu.umi.ac.ma (Z. Oumekloul).
} 
improve the performance and sensitivity of future nanosensors, but also to control them by changing the settings of the geometry, and the nature and thickness of the dielectric deposited on the structure for better quality detection.

This paper is organized as follows: In section 2 we present the technique of the formation of gold nanowires on glass and covered by $\mathrm{Si}_{3} \mathrm{~N}_{4}$ or $\mathrm{SiO}_{\mathrm{x}}$. Section 3 briefly the Lorentz - Drude model. Section 4 present the optical characterization of the structure and simulation. Sections 5, 6 and 7 study the effects of the geometrical parameters of the $\mathrm{Au}$ nanowires and dielectric layers on the plasmonic responses, respectively. The conclusion is presented in section 8 .

\section{Experimental section}

\subsection{Formation of gold nanostructures on glass}

Glass slides $(n=1.51)$ were cleaned in isopropanol and acetone in an ultrasound bath at room temperature, rinsed copiously with Milli-Q water, and dried under a stream of nitrogen. The clean substrates were transferred into an evaporation chamber. Gold nanostructures deposition was carried out by thermal evaporation of $4 \mathrm{~nm}$ thick gold films using MEB 550 S. Postdeposition annealing of the Au-covered slides was carried out at $500{ }^{\circ} \mathrm{C}$ for $1 \mathrm{~min}$ under nitrogen atmosphere, using a rapid thermal annealer (Jipelec Jet First 100). The reproducibility of the Au evaporation was evaluated by measuring the LSPR signals of a batch of 8 samples. The standard deviation in the wavelength $\left(\lambda_{R}\right)$ and maximum absorption $\left(I_{\max }\right)$ is typically $2 \mathrm{~nm}$ and 0.02 abs units, respectively.

\subsection{Deposition of dielectric overlayers}

\subsection{1. $\mathrm{SiO}_{x}$}

$\mathrm{SiO}_{x}$ overlayers were deposited on glass coated with $\mathrm{Au}$ nanostructures (glass/Au NWs) by plasma-enhanced chemical vapor deposition in a Plasmalab 800 Plus at a pressure of 0.005 Torr for $1 \mathrm{~h}$. The growth conditions used were as follows: substrate temperature, $300{ }^{\circ} \mathrm{C}$; gas mixture, $\mathrm{SiH}_{4}\left(5 \%\right.$ in $\mathrm{N}_{2}$ ) and $\mathrm{N}_{2} \mathrm{O}$ (the gas flow was 150 and 700 sccm for $\mathrm{SiH} 4$ and $\mathrm{N} 2 \mathrm{O}$, respectively); total pressure in the reactor, 1 Torr; and power, 20 Watt at $13.56 \mathrm{MHz}$. Under these experimental conditions, the deposition rate was $68.1 \mathrm{~nm} / \mathrm{min}$ and the silica films display a refractive index $n=1.45$. A total thickness of $300 \mathrm{~nm}$ was deposited and the thinner films were obtained by etching the $\mathrm{SiO}_{x}$ layer.

\subsection{2. $\mathrm{Si}_{3} \mathrm{~N}_{4}$}

$\mathrm{Si}_{3} \mathrm{~N}_{4}$ overlayers were deposited on glass coated with $\mathrm{Au}$ nanostructures by plasma-enhanced chemical vapor deposition in a Plasmalab 800Plus. The growth conditions used were as follows: substrate temperature, $200{ }^{\circ} \mathrm{C}$; gas mixture of $\mathrm{SiH}_{4}\left(5 \%\right.$ in $\left.\mathrm{N}_{2}\right)$ at $360 \mathrm{sccm}$ gas flow, $\mathrm{NH}_{3}$ at $20 \mathrm{sccm}$; total pressure in the reactor 1 Torr; power:
$10 \mathrm{~W}$ at $13.56 \mathrm{MHz}$. Under these experimental conditions, the deposition rate is $7.2 \mathrm{~nm} / \mathrm{min}$ and the films display a refractive index of $n=2.01$. A total thickness of $300 \mathrm{~nm}$ was deposited and the thinner films were formed by etching of the $\mathrm{Si}_{3} \mathrm{~N}_{4}$ layer.

\subsubsection{Etching of the $\mathrm{Si}_{3} \mathrm{~N}_{4}$ and $\mathrm{SiO}_{x}$ layers}

The $\mathrm{Si}_{3} \mathrm{~N}_{4}$ and $\mathrm{SiO}_{x}$ films are etched by a reactive ion etching (RIE) process using a gas mixture plasma in a RIE etching Plasmalab 80plus equipped with a laser interferometer (Jobin Yvon) at $670 \mathrm{~nm}$. The etching rate was calibrated using two separate techniques. First, the etching rate was estimated within the plasma chamber by an interferometer on $\mathrm{Si}_{3} \mathrm{~N}_{4}$ and $\mathrm{SiO}_{x}$ layer on plane silicon. Then, the etching rate was estimated by ellipsometry measurements. The etching rate is $48 \mathrm{~nm} / \mathrm{min}$ for $\mathrm{Si}_{3} \mathrm{~N}_{4}$ using the following etching parameters: gas mixture $\mathrm{SF}_{6}$ and $\mathrm{Ar}$ at 20 and $10 \mathrm{sccm}$ gas flow, respectively, $50 \mathrm{~W}$ forwarded power, and 10 mTorr chamber pressure. The etching rate is $27.2 \mathrm{~nm} / \mathrm{min}$ for $\mathrm{SiO}_{x}$ using the following etching parameters: gas mixture $\mathrm{CHF}_{3}$ and $\mathrm{CF}_{4}$ at $20 \mathrm{sccm}$ gas flow each, $180 \mathrm{~W}$ forwarded power, and 50 mTorr chamber pressure.

\subsection{Instrumentation}

\subsubsection{UV-vis spectrometer}

Absorption spectra were recorded with a Perkin-Elmer Lambda UV/ vis 950 spectrophotometer in cuvettes of $10 \mathrm{~mm}$ dimension. The wavelength range was $400-800 \mathrm{~nm}$.

\subsubsection{Scanning electron microscopy (SEM)}

SEM images were obtained with an electron microscope ULTRA 55 (Zeiss) equipped with a thermal field emission emitter and a high-efficiency In-lens SE detector.

\subsubsection{Ellipsometry}

Spectroscopic ellipsometry data in the visible range were obtained with a UVISEL Jobin Yvon Horiba Spectroscopic Ellipsometer equipped with DeltaPsi 2 data analysis software. The system acquired a spectrum ranging from 2 to $4.5 \mathrm{eV}$ with $0.05 \mathrm{eV}$ intervals. Data were obtained using an angle of incidence of $70^{\circ}$ with the compensator set at $45^{\circ}$ and fitted by regression analysis to a film-on-substrate model as described by their thickness and their complex refractive indices.

\section{Theoretical model}

Calculations are performed using the finite element method (FEM), which solves Maxwell's equations by discretizing space. Our calculation is performed in a two-dimensional (2-D) box (along $x$ and $y$ axes) with a propagation along the $y$ axis. Perfect matching layer (PML) conditions are applied at the boundaries $y$ of the box, to avoid reflections of outgoing waves. Along the $x$ direction, the unit cell is repeated periodically
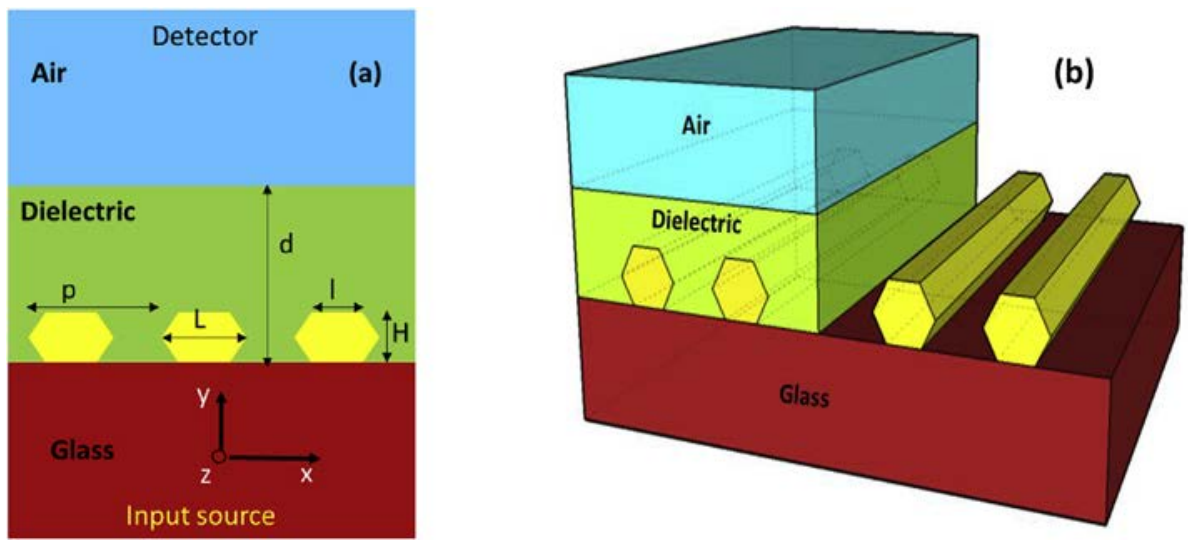

Fig. 1. (a) Schematic representation of the structure studied in this work. The gold nanowire is characterized by the height $H$ and the diameters $L$ and $l$. The inter-nanowire distance " $p$ " is defined as the distance between two nearest neighboring gold nanowires. The input source is placed in the glass substrate and the detector in air. (b) 3D model of the plasmonic nanostructure. (For interpretation of the references to color in this figure legend, the reader is referred to the Web version of this article.) 
(see Fig. 1) and the structure is supposed to be infinite along the $z$ direction. Space is discretized in both $x$ and $y$ directions using a triangular mesh.

The input electromagnetic field, having TM polarization, is generated at the left part of the unit cell by a current source parallel to the $x$ axis and having a planar profile along the $x$ direction. The transmitted signal, probed at the end of the right part of the unit cell, is recorded as a function of frequency. All the transmission spectra are normalized with respect to the one corresponding to a structure without the array of metallic nanoparticles.

The plasmonic nanostructure studied in this word is constructed by the gold nanowires deposited on a dielectric substrate and the latter are covered by air (see Fig. 1). The metal of dielectric constant is described by the Lorentz-Drude model for described the frequency-dependent complex permittivity of metal (gold) the formula is given by Ref. [33]:

$\varepsilon(\omega)=\varepsilon_{\infty}+\sum_{m=0}^{M} \frac{f_{m} \omega_{p}^{2}}{\omega_{m}^{2}-\omega^{2}+i \omega \Gamma_{m}}$

where $\varepsilon_{\infty}$ is the relative permittivity at infinite frequency, $\omega_{p}$ is the plasma frequency, $\omega_{m}, f_{m}$, and $\Gamma_{m}$ are, respectively, the resonance frequency, the strength, and the damping frequency of the mth oscillator. The Lorentz-Drude model uses $M=5$ damped harmonic oscillators to describe the small resonances observed in the metal's frequency response. The values of the constants in Eq. (1) are given in Table 1. This model allows us to fit experimental data for the frequency-dependent dielectric constant of metals such as gold [33] including both the real and imaginary parts, with good agreement in the visible wavelength range $(400<\lambda<800 \mathrm{~nm})$.

\section{Optical characterization of the structure and simulation}

Fig. 1 displays the schematic illustration of the sensor configuration used for the theoretical studies. A layer of gold nanoparticles of diameters $L$ and $l$, height $h$, and interparticle distance $p$ are deposited on a transparent glass substrate $\left(n_{\mathrm{SiO}_{2}}=1.51\right)$, coated with dielectric films of either $\mathrm{SiO}_{x}\left(n_{\mathrm{SiO}_{x}}=1.45\right)$ or $\mathrm{Si}_{3} \mathrm{~N}_{4}\left(n_{\mathrm{Si}_{3} \mathrm{~N}_{4}}=2.01\right)$ and covered with a nonabsorbing medium such as air with $n_{\text {air }}=1$.

The hexagonal gold nanowire (NW) used in this nanostructure is characterized by its height $\mathrm{H}$, the two diameters $\mathrm{L}$ and $l$, as well as the period p (see Fig. 1). During the study, we take into account a normal incidence of the incident electromagnetic field on the type of excitation, and we vary the wavelength in the visible range in order to detect the LSPR at the metal structure by modifying one of the previously defined geometrical parameters. Käll et al. [34], studied the LSPR frequency variation in function of the thickness of layers of tricosenoic acid 22 (a dielectric with a refractive index $n=1.53$ ). In this study, the thickness of the acid tricosenoic-22 deposited on the considered nanostructures varies from 0.0 to $340 \mathrm{~nm}$ [34]. The layers of dielectric are deposited on a $\mathrm{SiO}_{2}$ substrate, upon which two types of nanostructures have been studied experimentally. A random network of metallic gold nano-discs then a random network of air nano-holes perforated in a gold film with a thickness of $20 \mathrm{~nm}$, deposited on $\mathrm{SiO}_{2}$. A similar study was carried out with gold nanostructures deposited on $\mathrm{SiO}_{2}$ substrate. The layers of dielectric deposited on the metallic nanoparticles were $\mathrm{SiO}_{x}$ material

Table 1

The gold parameter values for Drude - Lorentz model [23].

\begin{tabular}{lllll}
\hline Oscillateur $\mathrm{m}$ & $\boldsymbol{f}_{\boldsymbol{m}}$ & $\omega_{\boldsymbol{p}}\left(\boldsymbol{r a d} . \boldsymbol{s}^{-1}\right)$ & $\omega_{\boldsymbol{m}}\left(\boldsymbol{r a d} . \boldsymbol{s}^{-1}\right)$ & $\boldsymbol{\Gamma}_{\boldsymbol{m}}\left(\boldsymbol{r a d} . \boldsymbol{s}^{-1}\right)$ \\
\hline 0 & 0.76 & $1.37188 .10^{16}$ & 0 & $8.05202 .10^{13}$ \\
1 & 0.024 & $1.37188 .10^{16}$ & $6.30488 .10^{14}$ & $3.66139 .10^{14}$ \\
2 & 0.01 & $1.37188 .10^{16}$ & $1.26098 .10^{15}$ & $5.24141 .10^{14}$ \\
3 & 0.71 & $1.37188 .10^{16}$ & $4.51065 .10^{15}$ & $1.32175 .10^{15}$ \\
4 & 0.601 & $1.37188 .10^{16}$ & $6.53885 .10^{15}$ & $3.78901 .10^{15}$ \\
5 & 4.384 & $1.37188 .10^{16}$ & $2.02364 .10^{16}$ & $3.36362 .10^{15}$
\end{tabular}

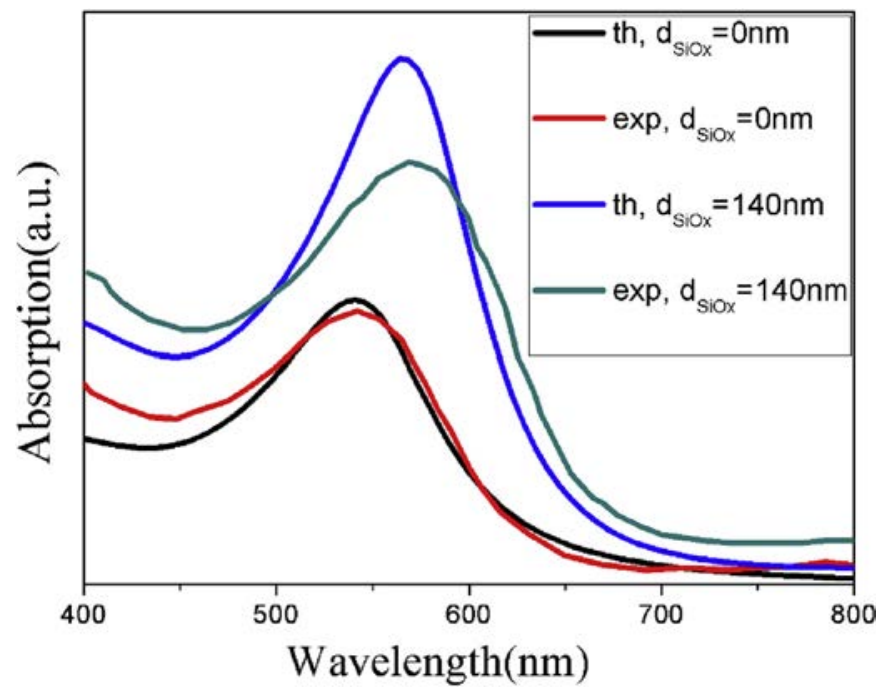

Fig. 2. Experimental and theoretical absorption spectra recorded on a glass/Au NWs interface without (red and black lines) and with a $140 \mathrm{~nm}$ thick $\mathrm{SiO}_{x}$ layer coating (blue and green). Parameters used for model: $l=28 \mathrm{~nm}, \mathrm{~L}=33 \mathrm{~nm}$, $\mathrm{p}=80 \mathrm{~nm}$ and $\mathrm{H}=15 \mathrm{~nm}$. (For interpretation of the references to color in this figure legend, the reader is referred to the Web version of this article.)

[35], and their thicknesses ranged from 0.0 to $300 \mathrm{~nm}$. The displacement of the observed properties of LSPR peaks in this study showed a red shift which was used for a study of the hybridization of the DNA molecule. These results showed the simultaneous influence of the dielectric refractive index deposited on the nanoparticles and its thickness on the behavior of the optical response at the interface LSPR. Galopin et al. have recently made the same comparison by using rectangular nanorods 2D [13]. Based on their experience, one can note a rather random particle shape, which requires them to determine statistical uncertainty with the dimensions of the nanoparticles in the overall structure. Akjouj et al. found that the most probable values for $l$ and $a$ respectively $25 \pm 8 \mathrm{~nm}$ in diameter and 16 to $8 \mathrm{~nm}$ inter-particle distance. For the third dimension which is the height $\mathrm{H}$ of the values obtained is $13.6 \pm 3 \mathrm{~nm}$ [36]. However, to keep the shape of our hexagonal structure, requires that the parameter $\mathrm{L}$ is larger than 1 , we took in this case $\mathrm{L}=1+5 \mathrm{~nm}$. The parameters selected for our structure resulting from these statistical results and the values chosen are: $l=28 \mathrm{~nm}$, $\mathrm{L}=33 \mathrm{~nm}, \mathrm{p}=80 \mathrm{~nm}, \mathrm{H}=15 \mathrm{~nm}$.

In Fig. 2, we compare the case where the thickness of the dielectric $\mathrm{SiO}_{x}$ deposited upon the metallic gold structures, whose thickness is $\mathrm{d}=140 \mathrm{~nm}$, as well as in the case where the gold metallic structure are in direct contact with air (no dielectric $\mathrm{SiO}_{x}$ ) whose thickness is $\mathrm{d}=0 \mathrm{~nm}$. We note a strong absorption around $\lambda_{R}=545 \mathrm{~nm}$ for both experimental and theoretical peaks, this value corresponds to the wavelength resonance that is to say, the plasmons excitation mode. Furthermore, one can grant the slight shift in magnitude to the sensitivity of the structure due to better sensitivity of hexagonal structure. However, we can decrease or increase the theoretical amplitude by adjusting it in function of the structure parameters, allowing to the experimental and theoretical results to be very close. In the second case we note a strong absorption in the vicinity of the wavelength resonance $\lambda_{R} \simeq 568 \mathrm{~nm}$ for both theoretical and experimental peaks, this value shows a shift towards the higher wavelengths of the absorption peak (almost a displacement of $23 \mathrm{~nm}$ in the visible band for a thickness of $140 \mathrm{~nm}$ ) compared to case where $d=0 \mathrm{~nm}$. This shows sensitivity of the structure to the displacements of the absorption peak for dielectric environment. The results obtained show a good agreement in terms of wavelength resonance. However there is a small difference in amplitude and the width of the peaks, which is due to a number of considerations including the comparison of a real system consisting of particles with 

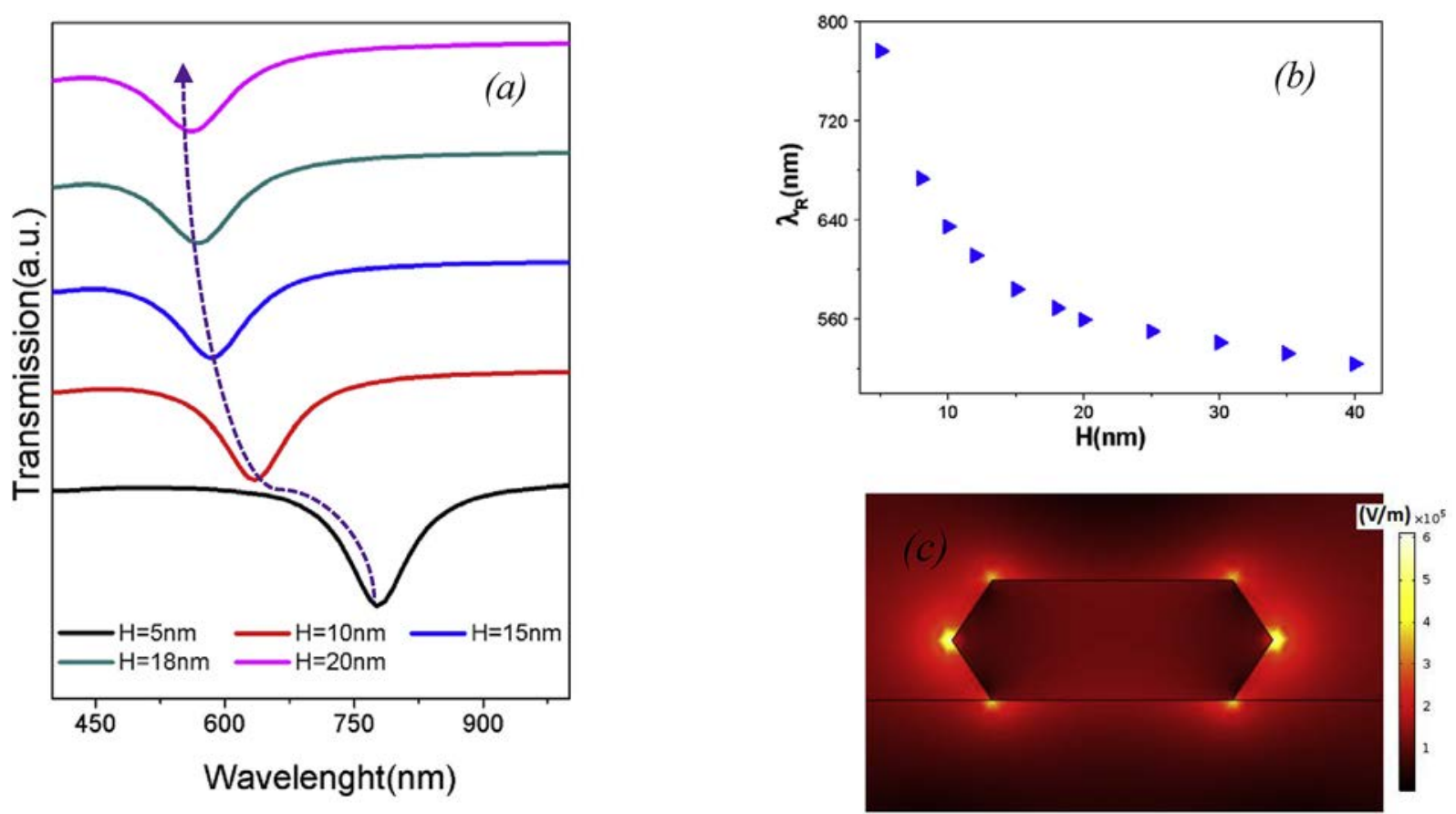

Fig. 3. (a) The transmission coefficient for a thickness $\mathrm{d}=140 \mathrm{~nm}$ of SiOx deposited on the gold hexagonal structure (dielectric constant calculated by the DrudeLorentz model) equal for TM polarization. (b) Evolution of LSPR $\lambda_{R}$ peak versus the heig H. (c) The field map show the distribution of the complex electric field amplitude for the resonance appearing in the spectrum for $\mathrm{H}=15 \mathrm{~nm}\left(\lambda_{R}=584.07 \mathrm{~nm}\right)$. (For interpretation of the references to color in this figure legend, the reader is referred to the Web version of this article.)

unequal sizes and ready for various use in a 3D simulation performed in 2D model described by a metal structure hexagonal according to the model of Drude-Lorentz.

\section{Influence of the geometrical parameters on plasmons response}

The modification of the geometric parameters of the metal structure (hexagonal) causes a change in the optical response of these nanoparticles. In this paragraph, we will focus on the changes caused by these geometric parameters. We admit the following values: $l=30 \mathrm{~nm}$, $\mathrm{L}=40 \mathrm{~nm}, \mathrm{p}=80 \mathrm{~nm}, \mathrm{H}=15 \mathrm{~nm}$, and each time, a parameter is varied, we keep the values of the others constant.

\subsection{The influence of the parameter $H$}

Fig. 3-a shows the variation of the transmission coefficient for $\mathrm{H}=5,10,15$ and $18 \mathrm{~nm}$ with a thickness of $\mathrm{SiOx} \mathrm{d}=140 \mathrm{~nm}$ deposited on the gold hexagon. Calculations Data: $\mathrm{L}=40 \mathrm{~nm}, 1=30 \mathrm{~nm}$, $\mathrm{p}=80 \mathrm{~nm}$, the substrate index $\mathrm{n}=1.51$, (constant thickness equal to $140 \mathrm{~nm}$ ).

We noticed a displacement to toward lower wavelengths with increasing $\mathrm{H}$, accompanied by a decrease in the amplitude which shows high sensitivity for low heights especially for $\mathrm{H}=5 \mathrm{~nm}$ (almost $-7 \mathrm{~dB}$ ). The minimum of the transmission coefficient corresponds to the resonance wavelength at which the incident wave is coupled to free electrons of metal particles, creating plasmons at the interface $\mathrm{SiO}_{2} / \mathrm{Au}$ / $\mathrm{SiO}_{x}$.

Fig. 3(b) shows changing the plasmons resonant wavelength of the particles according $\mathrm{H}$. A strong quasi-hyperbolic decay is observed in the resonance wavelength from $780 \mathrm{~nm}$ to $560 \mathrm{~nm}$ when the height of particles vary from $5 \mathrm{~nm}$ to $20 \mathrm{~nm}$, and it continues to be smaller but significant for a variation of the wavelength from $560 \mathrm{~nm}$ to $525 \mathrm{~nm}$ when $\mathrm{H}$ ranges from $20 \mathrm{~nm}$ to $40 \mathrm{~nm}$.

\subsection{The influence of the parameter $p$}

In this section, we will study the influence of the frequency p over plasmonic resonance by calculating the transmission coefficient for TM polarization. The period of structure is a very important parameter as it depends on the resonance related to the excitation of surface plasmons and describes the dipolar interactions in between particles at nanometre's level.

The calculation data are: $\mathrm{L}=40 \mathrm{~nm}, 1=30 \mathrm{~nm}, \mathrm{H}=15 \mathrm{~nm}$ for $\mathrm{p}=45,50,60,70,80$ and $90 \mathrm{~nm}$. It is therefore possible to see the influence of the geometry of the structure, by varying $\mathrm{p}$, one can easily see the effect on the transmission spectrum for a TM polarization. Fig. 4(a) presents the transmission spectrum versus the wavelength. Gradually, as the particles approach each other, the optical behavior of structure becomes increasingly sensitive; when the particles approach, $\mathrm{p}=45 \mathrm{~nm}$, it shows a strong absorption of incident energy $(-15 \mathrm{~dB})$. This indicates that each dipole (metal structure) is subjected to an electric field resulting from the sum of the incident field and local fields produced by each of the neighboring dipole, and when the inter-particle distance is small, the mutual influence of particles has a strong coupling which makes the area more sensitive, for a better detection. The symmetric and homogenous charge distributions on both the left and right borders of the nanoparticle (see below Fig. 4 (a)) represent a quite evident consequence of the mode excitation for $\mathrm{p}=45 \mathrm{~nm}$ induced by the TM wave.

There is a drop in the resonant wavelength from $720 \mathrm{~nm}$ to $582 \mathrm{~nm}$ (138 nm decrease), when p vary from $45 \mathrm{~nm}$ to $80 \mathrm{~nm}$ [see Fig. 4(b)]. Then there is a low decrease, from $582 \mathrm{~nm}$ to $580 \mathrm{~nm}$, where p ranges from $80 \mathrm{~nm}$ to $90 \mathrm{~nm}$. For low coupling between the metallic nanostructures, $\mathrm{p}$ variation has almost no effect on the response of the particles. So, compared to other structures considered as the gold ribbon a better result can still be seen. This structure concentrates and absorbs the field in a very sub-wavelength volume, which represents a crucial interest for detection. Fig. 4(c) represents the electric field distribution at the incident wavelengths (of the dips) $\lambda_{R}=718 \mathrm{~nm}$. At these wavelengths, the field is trapped in the nanoparticle cavity and does not 

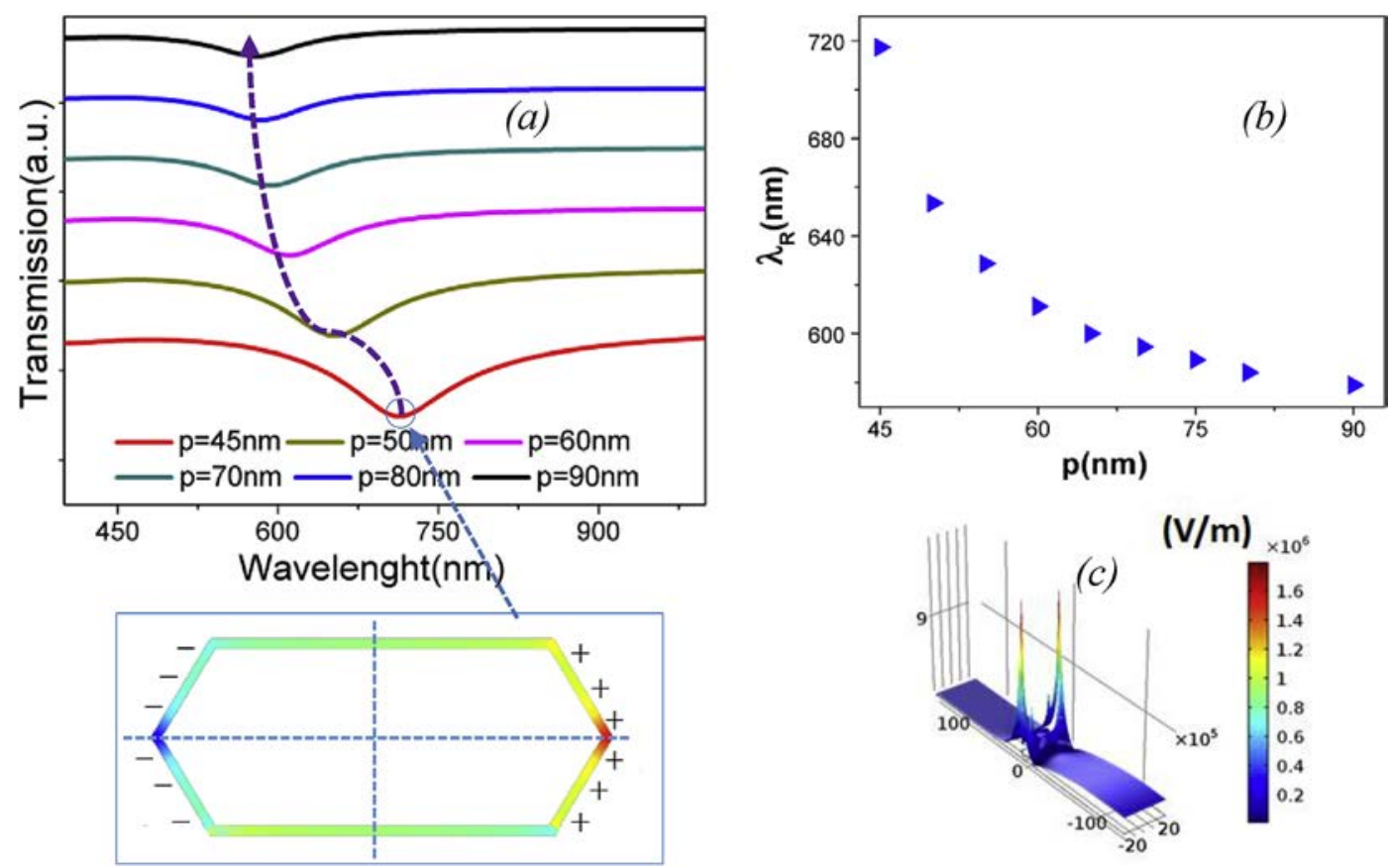

Fig. 4. (a) Transmission coefficient for a thickness $\mathrm{d}=140 \mathrm{~nm} \mathrm{SiO}$ deposited on hexagonal gold structure (dielectric constant calculated by the Drude-Lorentz model) equal for TM polarization with surface charge of dipolar mode at resonance frequency $\left(\lambda_{R}=717.9 \mathrm{~nm}\right)$. (b) Evolution of the resonance wavelength as a function of $\mathrm{p}$. (c) Presents the electric field map at $\mathrm{p}=45 \mathrm{~nm}\left(\lambda_{R}=717.9 \mathrm{~nm}\right)$. (For interpretation of the references to color in this figure legend, the reader is referred to the Web version of this article.)

propagate in the system.

\subsection{The influence of the parameter $L$}

The effect of parameter $\mathrm{L}$ will be observed in the transmission spectrum for TM polarization while keeping these constant parameters: $\mathrm{H}=15 \mathrm{~nm}, \mathrm{~L}=35 \mathrm{~nm} \mathrm{p}=80 \mathrm{~nm}$ for $\mathrm{L}=40,45,50,55,60$ and $65 \mathrm{~nm}$.
From the transmission spectrum (Fig. 5-a) an increase in the resonant wavelength was observed towards higher wavelengths (toward red) when increasing L. The movement takes place from $600 \mathrm{~nm}$ to $759 \mathrm{~nm}$ for a progressive increase of $L=40 \mathrm{~nm}-65 \mathrm{~nm}$. This means an increase in variation of $150 \mathrm{~nm}$ for an increase of $\mathrm{L}=25 \mathrm{~nm}$. We also note a strong absorption, more specifically for $\mathrm{L}=65 \mathrm{~nm}$ (almost $-14 \mathrm{~dB}$ ), that can be connected to the energy containment in the presence of a
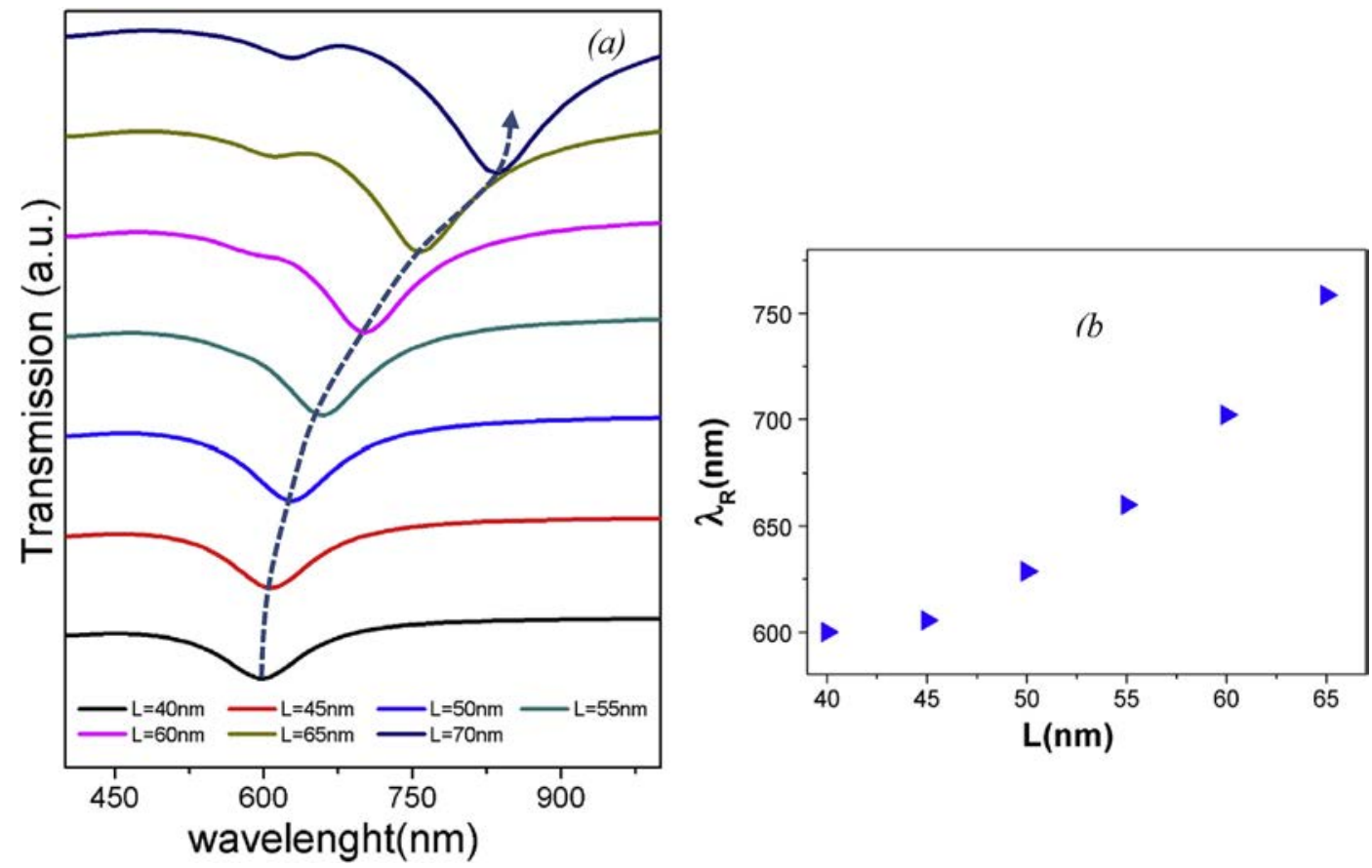

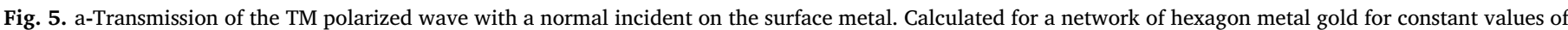
$\mathrm{H}=15 \mathrm{~nm}, \mathrm{l}=35 \mathrm{~nm}$ and $\mathrm{p}=80 \mathrm{~nm}$ b- Evolution of the resonance wavelength as a function of L. (For interpretation of the references to color in this figure legend, the reader is referred to the Web version of this article.) 

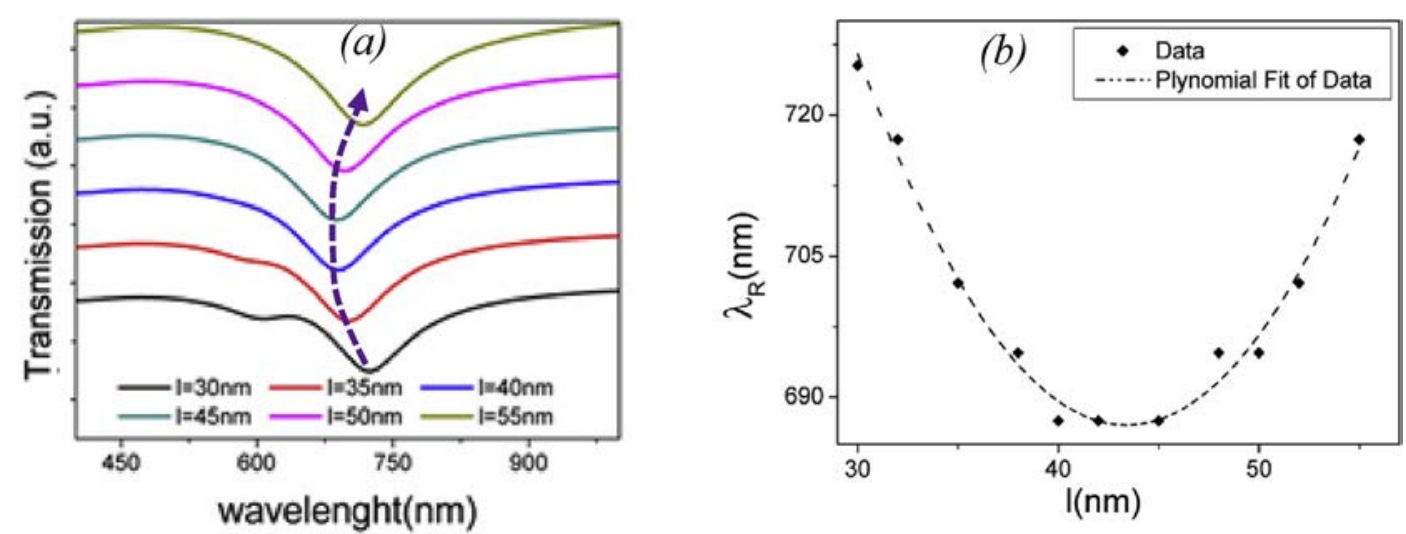

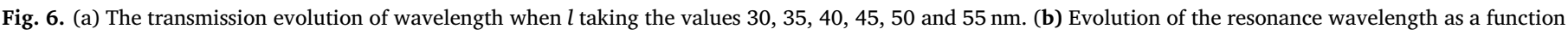
of $l$.

large exchange surface on the dielectric/metal interface. Starting from $60 \mathrm{~nm}$, we observe the appearance of another peak around $600 \mathrm{~nm}$. The latter appeared due to the change of the charging orientation of the dipolar mode.

Fig. 5-b shows the evolution of the resonance wavelength depending on $\mathrm{L}$, which links it directly to the behavior of the resonance wavelength of the plasmons. Indeed, it is noticed, between $40 \mathrm{~nm}$ and $50 \mathrm{~nm}$, that the plasmon resonance wavelength is growing a non-linear manner with L. Between $50 \mathrm{~nm}$ and $60 \mathrm{~nm}$, of the plasmon resonance is growing almost linearly with $\mathrm{L}$, which shows a reasonable agreement with the result obtained for the parameter $\mathrm{p}$, since by increasing $\mathrm{L}$, nanostructures approach and coupling remain increasingly strong, leading on the one hand to a high sensitivity. On the other hand to a movement toward the infrared, which presents an advantage to the biosensor in the infrared range.

\subsection{The influence of the parameter $l$}

Likewise, it is possible to study the influence of the transmission curve (Fig. 6) for TM polarization. Thus, the choice of the other parameters is given by: $\mathrm{L}=60 \mathrm{~nm}, \mathrm{H}=15 \mathrm{~nm}, \mathrm{p}=80 \mathrm{~nm}$ for the values $\mathrm{L}=30,35,40,45,50$ and $55 \mathrm{~nm}$.

Fig. 6(a) shows that parameter $l$ has great influence on the peaks amplitude, because the displacement does not matter between the values. There is a displacement of $23 \mathrm{~nm}$ for $l=30 \mathrm{~nm}-35 \mathrm{~nm}, 14.5 \mathrm{~nm}$ for $l=35 \mathrm{~nm}-40 \mathrm{~nm}, \quad 0 \mathrm{~nm}$ for $l=40 \mathrm{~nm}-45 \mathrm{~nm}, \quad 7.5 \mathrm{~nm}$ for $l=45 \mathrm{~nm}-50 \mathrm{~nm}$ and $22 \mathrm{nml}=50 \mathrm{~nm}-55 \mathrm{~nm}$. For $l=30 \mathrm{~nm}$ and $55 \mathrm{~nm}$, we have almost the same resonance wavelength $(725 \mathrm{~nm}$ and $717 \mathrm{~nm}$ respectively), but the second value is better in terms of amplitude. Fig. 6(b) shows the evolution of 1 on the resonance wavelength of each peak as a function of $l$.

Based on Fig. 6(b) an almost linear decrease is observed between $l=30 \mathrm{~nm}$ to $40 \mathrm{~nm}$, for this interval, the peaks move to the short lengths wave and remain constant between $40 \mathrm{~nm}$ and $45 \mathrm{~nm}$ then grow to larger wavelengths from $45 \mathrm{~nm}$ to $55 \mathrm{~nm}$. As for the amplitude, there is a gradual improvement due to the width of $l$ which is in direct contact with the dielectric making better sensitivity for this hexagonal structure.

According to the observation of the various figures, we see that the resonance shape peaks and the value of the resonant frequency evolve according to the geometric parameters choice of the hexagonal structure. This give better results for the sensitivity of biosensors.

\section{Effect of dielectric layers on the plasmons response}

In sections 6.1 and 6.2 of this part, we will focus on the effect of the refractive index and the thickness of dielectric medium deposited on top of the metal structure. The two dielectrics studied are silicon oxide
$\mathrm{SiO}_{x}$ whose refractive index is $n_{\mathrm{SiO}}=1.45$ and the silicon nitride $\mathrm{Si}_{3} \mathrm{~N}_{4}$ whose refractive index is $n_{S_{3} N_{4}}=2.01$. They are widely used experimentally for their poor absorption and can protect nanoparticles from morphological changes when applied to aqueous solutions or organic solvents. The choice of these two dielectric is not arbitrary that is why we will study them:

$\checkmark$ The refractive index of the substrate is higher than that of the dielectric layer $\left(\boldsymbol{n}_{\mathrm{SiO}_{2}}>\boldsymbol{n}_{\mathrm{SiOx}}\right)$.

$\checkmark$ The substrate refractive index is lower than that of the dielectric layer filed $\left(\boldsymbol{n}_{\mathrm{SiO}_{2}}<\boldsymbol{n}_{\mathrm{Si}_{3} \mathrm{~N}_{4}}\right)$.

We used the following silicon oxide alloys and silicon nitride, deposited on metallic gold nanostructures immobilized to the glass, to study the behavior of the plasmons response and choose the best dielectric and the thickness to enhance the sensitivity of detection of the biosensor.

\subsection{The effect of $\mathrm{SiO}_{\boldsymbol{x}}$}

The effect of the thickness of $\mathrm{SiO}_{x}$ deposited on the hexagonal structure of gold is carried out when used in giving the thickness of the $\mathrm{SiO}_{x}$ layer the values $\mathrm{d}=20 \mathrm{~nm}, 80 \mathrm{~nm}, 120 \mathrm{~nm}, 160 \mathrm{~nm}, 280 \mathrm{~nm}$, while preserving the following parameters: $\mathrm{L}=40 \mathrm{~nm}, 1=30 \mathrm{~nm}$, $\mathrm{p}=80 \mathrm{~nm}$. Fig. 7 shows the curve of the transmission coefficient of an interface $\mathrm{SiO}_{x}$-Air for TM polarization.

There is an oscillation in the resonance wavelength for a range of value of the thickness of the dielectric with a quite good sensitivity. These oscillations observed in the spectrum are due to coupling between the plasmons mode and the modes of a Fabry-Perot cavity connected to the presence of the oxide layer silicon $\mathrm{SiO}_{x}$ presented above the gold nanowire chain: Light incoming perform multiple round-trip within the cavity defined by $\mathrm{SiO}_{x}$ and $\mathrm{SiO}_{2}$, and partially spring at each reflection.

\subsection{The effect of the $\boldsymbol{S i}_{3} \boldsymbol{N}_{4}$ dielectric overlayer thickness}

When changing the $\mathrm{SiO}_{x}$ dielectric by the chemical compound of formula $\mathrm{Si}_{3} \mathrm{~N}_{4}$, the plasmons response of gold nanoparticles changes. Silicon nitride is a hard ceramic whose physical properties are maintained over a wide temperature range. Fig. 8 (a) shows the evolution of the transmission spectra versus wavelength of an interface $S_{3} N_{4}$ /Air, depending on the thickness of the $\mathrm{Si}_{3} N_{4}$ dielectric layers for values $\mathrm{d}=20 \mathrm{~nm}, 80 \mathrm{~nm}, 120 \mathrm{~nm}, 160 \mathrm{~nm}$ and $280 \mathrm{~nm}$, for TM polarization. The hexagonal gold nanowires have the following calculation parameters: $\mathrm{L}=40 \mathrm{~nm}, 1=30 \mathrm{~nm}, \mathrm{p}=80 \mathrm{~nm}$, with the index of the substrate $n_{\mathrm{SiO}_{2}}=1,51$.

An increase in the amplitude is deduced, so a strong absorption 


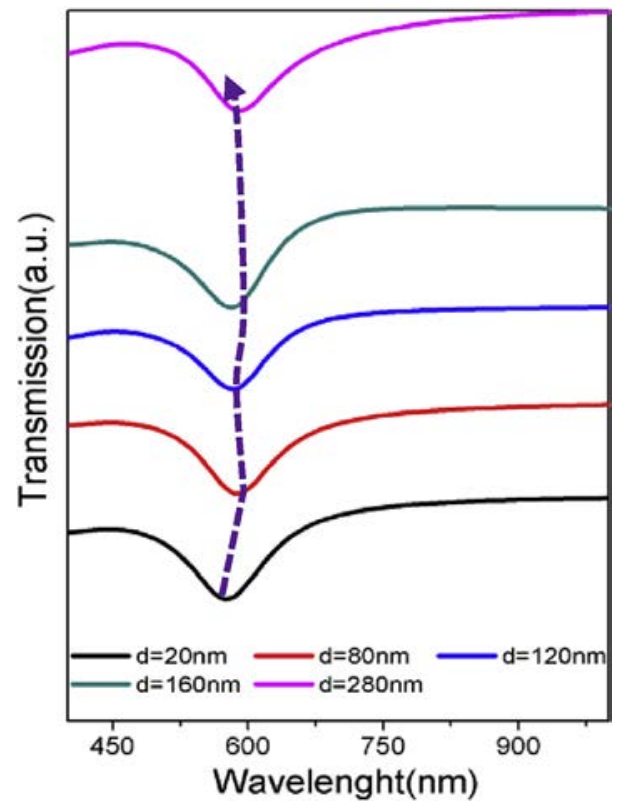

Fig. 7. Spectral shape and position of the LSPR peak of transmission depending on the thickness of $\mathrm{SiO}_{x}$ layer.

which improves the sensibility in an extraordinary way, which is doubled when compared to the $\mathrm{SiO}_{x}$ case, and also to the sensitivity of the structure studied in Fig. 8. Fig. 8(b) shows the change in $\lambda_{R}$ as a function of $\mathrm{Si}_{3} \mathrm{~N}_{4}$ thickness. It was predicted that the LSPR shift should saturate at $\Delta \lambda_{R} \approx m \Delta n \approx 46 n m[27,28,37]$, where $m$ is the refractive index sensitivity for the glass/Au NWs interface $\left(m=\frac{\delta \lambda_{R}}{\delta n}=90 \mathrm{~nm}\right)$ and $\Delta n$ the change in the refractive index induced by the absorbate. Van Duyne and co-workers explored experimentally the long-range distance dependence of LSPR nanosensors using self-assembled monolayers (SAMs) of 11-mercaptoundecanoic acid and $\mathrm{Cu}^{2+}$ ions absorbed on surface-confined noble metals nanoparticles [27]. It was found that the shift of $\lambda_{R}$ with increasing adsorbate thickness is nonlinear and has a sensing range that is dependent on the composition, shape, width, and height of the nanoparticles. The use of atomic layer deposition of $\mathrm{Al}_{2} \mathrm{O}_{3}$ enabled, in addition, the study of both the long- and short-range distance dependence of the LSPR nanosensors [37,38]. At short distances

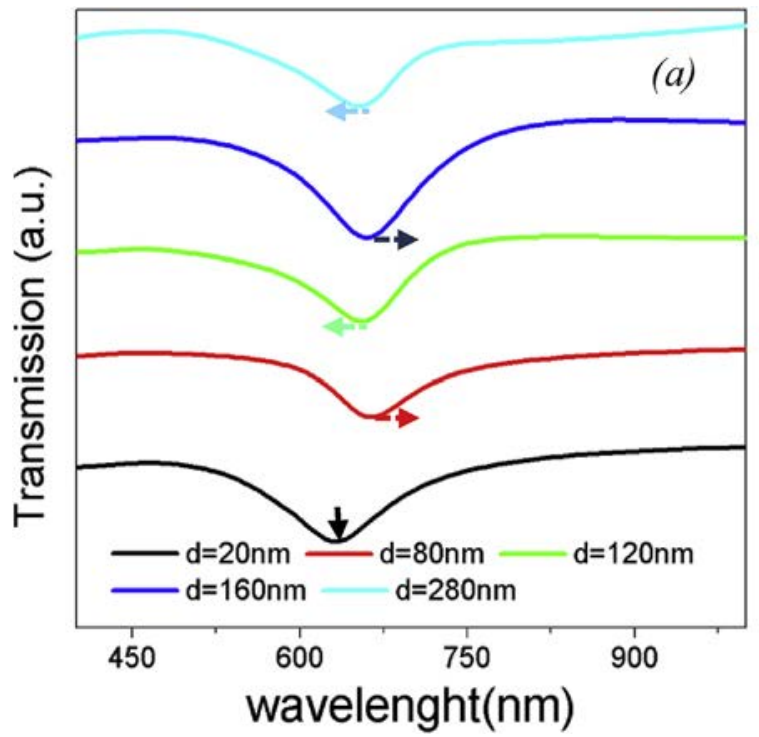

from the nanoparticles surface, the LSPR $\lambda_{R}$ shift follows a steep linear slope, but as the distance from the nanoparticles increases, the curve bends over and eventually levels off once a saturation point is reached. This is clearly not observed for the interfaces under study.

Fig. 8(b) shows that the presence of a $\mathrm{Si}_{3} \mathrm{~N}_{4}$ overlayer causes theoretically first a red shift until saturation at $d_{\mathrm{Si}_{3} \mathrm{~N}_{4}} \approx 62 \mathrm{~nm}$. In the range $62<d_{S_{3} N_{4}}<115 \mathrm{~nm}$ blue shift in $\lambda_{R}$ is observed, followed by a red shift until $d_{S_{3} N_{4}} \approx 230 \mathrm{~nm}$. This oscillation extends until $1080 \mathrm{~nm}$ with a periodicity of $d_{p}=168 \mathrm{~nm}$ and with increasing amplitude at $d_{\mathrm{Si}_{3} \mathrm{~N}_{4}}>850 \mathrm{~nm}$. The resonances (corresponding to the maximum of the amplitude of the oscillations) and the anti-resonances (corresponding to the minimum of the amplitude of the oscillations) are similar to the classical Fabry-Perot cavity. Moreover, the periodicity of the oscillation can be calculated by the equation $d_{p}=\lambda_{R} / 2 n$, where $n$ is the refractive index of the dielectric layer $\left[d_{p} \approx \frac{664}{2 \times 2.01}=165 \mathrm{~nm}\right.$ (see Fig. 8(b))]. The oscillation is anharmonic and the plasmon shift is faster in the blue-shifting regions (e.g., $90 \mathrm{~nm}, 255 \mathrm{~nm}$, etc.) than in the redshifting regions (e.g., $20 \mathrm{~nm}, 185 \mathrm{~nm}$, etc.). The results in Fig. 8(b) indicate that refractive index sensitivity increases with increasing distance (i.e., the particle are infinitely and increasingly more sensitive to layer thicknesses). Finally, let us notice that the dielectric deposited on the metallic gold nanostructure having a wide index relative to that of the substrate, leads to better results thanks to the sensitivity of the plasmonic response function of the thickness $d$. This structure absorbs the concentrated field in a volume with sub-wavelength, which represents a crucial interest for detection.

The calculated LSPR curves are corroborated by the experimental LSPR curves recorded on glass/Au nanowire coated with $\mathrm{SiO}_{\mathrm{x}}$ of increasing thicknesses. Fig. 9 shows the obtained shift in $\lambda_{R}$ together with the ones predicted through modeling. The square dots represent the theoretical results whereas the triangle-shaped dots corresponds to the experimental ones. For all the $\mathrm{Si}_{3} \mathrm{~N}_{4}$ overlayer, the theoretical results agree with the experimental one.

\section{Study of the sensitivity}

The plasmonics LSPR nano-sensors works by variation of the resonance wavelength. It is clear that the plasmonic response of metallic nanoparticles is strongly depend on their size and shape. In addition, this dependence affects the importance of the spectral shift of the resonance, following the change in the refractive index of the medium located near the metal nanowires. We showed a high sensitivity of the

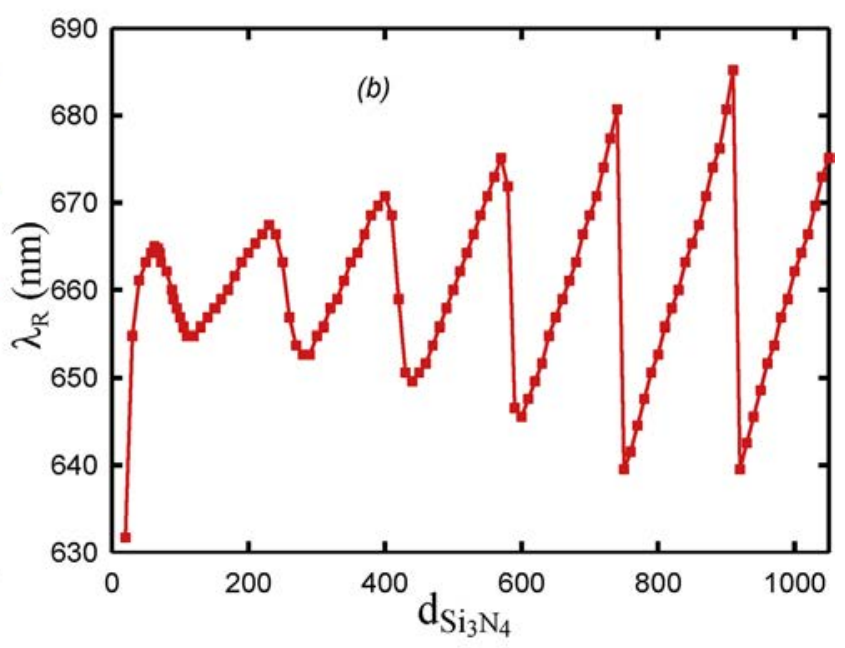

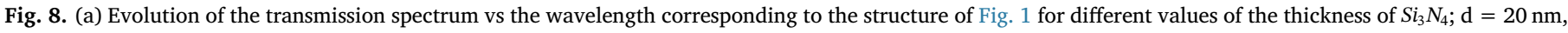
$80 \mathrm{~nm}, 120 \mathrm{~nm}, 160 \mathrm{~nm}, 280 \mathrm{~nm}$. (b) Oscillation of resonance wavelength. 


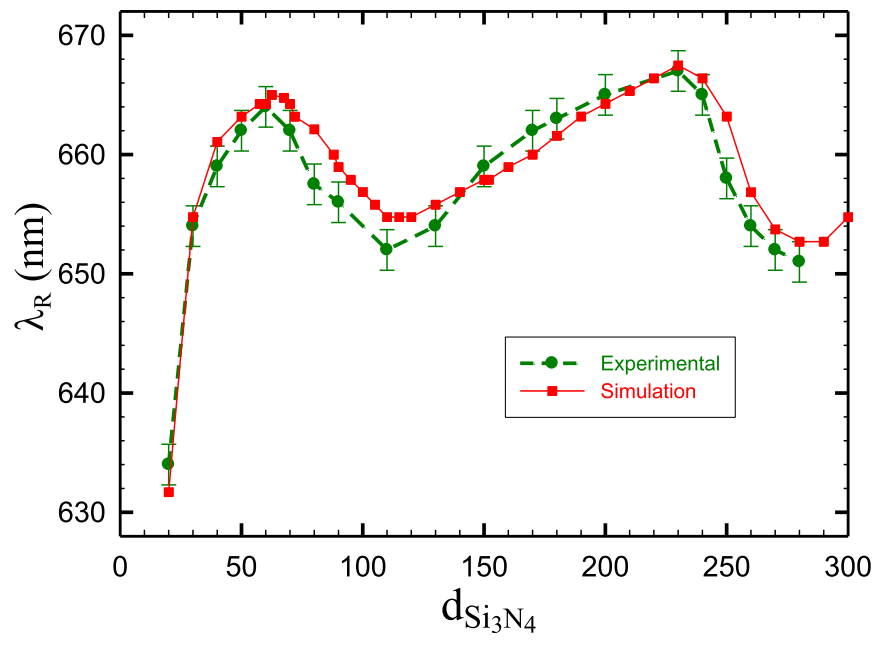

Fig. 9. Evolution of $\lambda_{R}$ with the thickness of the $\mathrm{Si}_{3} \mathrm{~N}_{4}$ overlayer; comparison of experimental (triangle-shaped dots) and theoretical (square dots) data.

metal nanoparticle plasmon response with different geometrical parameters of Au nanoparticles and/or refractive index of the dielectric deposited on the top of the Au nanoparticles. Fig. 1 show the proposed sensing structure, where the layer dielectric is on the top of Au nanowire. To qualify the performance of the proposed sensor, we compute a sensing $\mathrm{S}$. The expression used to calculate the sensitivity is given by:

$S=\frac{\Delta \lambda_{R}}{\Delta n}$,

where $\Delta n$ is the variation of the refractive index between the dielectric $\left(\mathrm{SiO}_{x}\right)$ and one of the other substance used and $\Delta \lambda_{R}$ the variation of the resonance wavelength.

In the first part, we investigate theoretically the influence of the refractive index of the overcoating relative to the underlying substrate, supporting the metal nanostructures, on the sensitivity of the multilayered interface for sensing. This is indeed a highly important parameter for the development of a sensitive LSPR interface. The structure studied here consists of gold nanowires of height $H=15 \mathrm{~nm}$ and the diameters $L=40 \mathrm{~nm}$ and $l=30 \mathrm{~nm}$ deposited on a $\mathrm{SiO}_{2}$ substrate and coated with dielectric film with $d=20 \mathrm{~nm}$ (see Fig. 1). The lattice parameter $p=80 \mathrm{~nm}$. Three different cases can thus be distinguished: (i) the refractive index of the substrate is higher than that of the deposited dielectric layer; (ii) the refractive index of the substrate is the same as that of the deposited dielectric layer; and (iii) the refractive index of the substrate is lower than that of the deposited dielectric layer. The interface is furthermore covered by a air. To obtained theses three case, we use the following dielectrics: $\mathrm{H}_{2} \mathrm{O}, \mathrm{C}^{6} \mathrm{H}_{6}, I T O$ and $\mathrm{Si}_{3} \mathrm{~N}_{4}$ and air as reference.

Table 2 presents a comparison of sensitivity between different dielectrics deposited on the Au nanowires. The thickness of the dielectric layer is $\mathrm{d}=20 \mathrm{~nm}$ in all case studied here. We noticed that benzene has a very high sensitivity; it can be explained by the fact that the benzene has the same refractive index as the substrate (glass). In other words, because of the fact that the refractive index are the same, there is an absence of the interface between the substrate and the dielectric. In this

Table 2

Comparison table in sensitivity between different substances for hexagonal gold nanoparticles.

\begin{tabular}{lll}
\hline Dielectric & Refractive index & Sensitivity (nm/RIU) \\
\hline Water & 1.33 & 249.5 \\
Benzene & 1.50 & 607 \\
ITO & 2.00 & 406.5 \\
$\mathrm{Si}_{3} \mathrm{~N}_{4}$ & 2.01 & 415.7 \\
\hline
\end{tabular}

case, the Fabry-Perot cavity mode disappears which is not the case for where the refractive index of the dielectric is different from the substrate refractive index [cases (i) and (iii)]. In the last case, when the refractive index of the over-layer is higher than substrate refractive index, the sensitivity is relatively good. In this situation, the oscillations cavity mode increases and coupled with plasmonic monde, however the existence of the interface between the substrate and the dielectric affects the sensibility because they reduce the confinement of the electric field supported by Au nanowires. From this model it emerged that the sensitivity exclusively depends on the LSPR band location and the refractive index of the dielectric. We conclude that the optimal sensitivity is obtained when the index of the overlayer is of the same nature as that the refractive index of the substrate.

In the second part, we compare the evolution of the sensitivity between different geometrical gold nanowires but with the same basic area $\left(1500 \mathrm{~nm}^{2}\right)$. The sensitivity of the structures considered has been determined by identifying the position of the main resonance mode on their transmission spectra when the middle of the detection is air and $\mathrm{Si}_{3} \mathrm{~N}_{4}$. The structure studied here consists of gold nanowires of height $H$ $=25 \mathrm{~nm}$ and the diameters $L=80 \mathrm{~nm}$ and $l=40 \mathrm{~nm}$ deposited on a $\mathrm{SiO}_{2}$ substrate and coated by dielectric film with thickness $d=30 \mathrm{~nm}$. In this study, we have used $\mathrm{Si}_{3} \mathrm{~N}_{4}$ as a dielectric because the sensitivity of nanoparticles situated in a homogeneous host matrix (i.e. this situation correspond to the case where the refractive index of the substrate and the dielectric is the same) is independent of their shape [38]. The sensibility was also approximated in this study by $\Delta \lambda_{L S P R} / \Delta n$. We observe that the nanowire with the base in hexagonal form present a greater sensitivity than the other geometric shapes of the nanowire. The results are summarized in Table 3.

The origin of the high sensitivity of the infrared plasmon band is due to the high electrical field confinement between the Au nanostructures and due to the shape of the nanostructures itself. The hexagonal structure supports a strong localization of the electric field in the corners of the gold NPs that are numerous in our structure compared to others (we may well see it in the mapping of the field), In fact, the sensitivity of a plasmonic structure is also dependent on the location of the field around the nanoparticles at the resonance. The hexagonal geometry has 6 hot spots, two of which are in contact with the substrate, which generally has losses in terms of localized field intensity. We can draw the following conclusion; the exaltation of the electric field in the neighborhood NPs improves the sensitivity of the device. Additionally, the choice of the covering layer, which is accompanied by an index contrast with the substrate, amplifies the confinement of the electrical field due to interference created by the Fabry-Perot cavity.

The theoretical results obtained show that hexagonal nanowires is two time more sensitive than a triangular nanowire, three time more than a circular nanowire and 2.3 more sensitive than rectangular nanowire (see Table 3). These results will improve the performance of future nano-biosensors.

\section{Conclusion}

We have developed a theoretical study of optical properties of hexagonal gold metal nanoparticles, deposited on a glass substrate,

Table 3

Comparison table in sensitivity between circular, rectangular, hexagonal and triangular gold nanowires (the basic area of the nanowire $=1500 \mathrm{~nm}^{2}$ )

\begin{tabular}{ll}
\hline Geometry & Sensitivity \\
\hline Circular $\bigcirc$ & $202.0 \mathrm{~nm} / \mathrm{RIU}$ \\
Rectangular $\square$ & $270.3 \mathrm{~nm} / \mathrm{RIU}$ \\
Triangular $\square$ & $307.0 \mathrm{~nm} / \mathrm{RIU}$ \\
Hexagonal $\square$ & $620.8 \mathrm{~nm} / \mathrm{RIU}$
\end{tabular}


$\mathrm{SiO}_{2}$, upon one of the dielectric, $\mathrm{SiO}_{x}$ or $\mathrm{Si}_{3} \mathrm{~N}_{4}$ by exploiting the resonance localized surface plasmons (LSPR) capable of detecting molecules at a nano-scale. In fact, the hexagonal structure is 2.3 times better than the rectangular because the sensitivity of the hexagonal is $621 \mathrm{~nm} /$ RIU, and thus way greater than the other structures. We have seen that the plasmons response of the hexagonal structure based on geometrical parameters showed better sensitivity than gold ribbon. This has improved the sensitivity of the interface, which allows us to have nanosensors very sensitive in order to be more adequate to the biological and chemical detection. It was also seen that the resonance wavelength of the plasmons response manifest an oscillatory variation in function of the thickness of dielectric layers deposited on the particles. This phenomenon is interpreted in terms of the coupling of mode surface plasmons particles with those of the optical cavity, which consists of $\mathrm{SiO}_{x}$, bounded on both sides of the detection medium and $\mathrm{SiO}_{2}$ substrate. The same result was shown with $\mathrm{Si}_{3} \mathrm{~N}_{4}$, which has a contrast index greater with $\mathrm{SiO}_{2}$ but slightly shifted towards longer wavelengths and with a better sensitivity. Significant efforts have been made experimentally in the last years to increase the sensitivity of the refractive index of the interface LSPR, but it seems necessary to wait a few years to see technical improvement, particularly with the new generation of detectors.

\section{Acknowledgments}

Z.O. and S.L. gratefully acknowledge the hospitality of the Institute of Electronics, Microelectronics and Nanotechnologies (IEMN), UMR CNRS 8520 and Department of Physics, University of Lille.

\section{References}

[1] M. Faraday, Experimental relations of gold (and other metals) to light, Phil. Trans. R. Soc. Lond. January 1 (1857).

[2] J. Li, C. Chen, L. Lagae, P. Van Dorpe, J. Phys. Chem. C119 (2015) 29116-29122.

[3] W.R. Erwin, R. Bardhan, J. Phys. Chem. C 120 (2016) 29423-29431.

[4] M. Focsan, A.M. Cracium, M. Poatara, C. Leordean, A. Vulpoi, D. Maniu, S. Astilean, Sci. Rep. 7 (2017) 14240.

[5] S.A. Maier, M.L. Brongersma, P.G. Kik, S. Meltzer, A.A.G. Requicha, H.A. Atwater, Plasmonics - a route to nanoscale optical devices, Adv. Mater. 13 (2001) 1501-1505.

[6] T. Abhilash, M. Balasurbrahmaniyam, A. Patra, S. Kasiviswanathan, Appl. Phys. Lett. 104 (2014) 1-5.

[7] S. Pillai, K.R. Catchpole, T. Trupke, M.A. Green, J. Appl. Phys. 101 (2007) 093105.
[8] K.R. Catchpole, A. Polman, Appl. Phys. Lett. 93 (2008) 191113.

[9] K.M. Mayer, J.H. Hafner, Chem. Rev. 111 (2011) 3828-3857.

[10] S. Szunerits, R. Boukherroub, Chem. Commun. 48 (2012) 8999-9010.

[11] O. Krupin, W.R. Wong, P. Beland, F.R.M. Adikan, P. Berini, J. Lightwave Technol. 34 (2016) 4673-4681.

[12] J. Zhang, L. Zhang, W. Xu, J. Phys. D Appl. Phys. 45 (2012) 113001.

[13] E. Galopin, J. Niedziolka-Jonsson, A. Akjouj, Y. Pennec, B. Djafari-Rouhani, A. Noual, R. Boukherroub, S. Szunerits, J. Phys. Chem. C 114 (2010) 11769-11775.

[14] E. Galopin, A. Noual, J. Niedziolka-Jonsson, M. Jonsson-Nedziolka, A. Akjouj, Y. Pennec, B. Djafari-Rouhani, R. Boukherroub, S. Szunerits, J. Phys. Chem. C 113 (2009) 15921-15927.

[15] S. Szunerits, S. Ghodbane, J. Niedziolka-Jonsson, E. Galopin, F. Klauser, A. Akjouj, Y. Pennec, B. Djafari-Rouhani, R. Boukherroub, D. Steinmuller-Nethl, J. Phys. Chem. C 114 (2010) 3346-3353.

[16] S. Szunerits, V.G. Praig, M. Manesse, R. Boukherroub, Nanotechnology 19 (2008) 195712-195719.

[17] N. Nath, A. Chilkoti, Anal. Chem. 76 (2004) 5370-5378.

[18] K.M. Mayer, S. Lee, H. Liao, B.C. Rostro, A. Fuentes, P.T. Scully, C.L. Nehl, J.H. Hafner, ACS Nano 2 (2008) 687-692.

[19] A.W. Murray, B. Auguié, L.W. Barnes, J. Phys, Chem. C 113 (2009) 5120-5125.

[20] J. Homola, Surface Plasmon Resonance Based Sensors, Springer Series on Chemical Sensors and Biosensor/Methods and Applications vol. 4, Springer-Verlag Berlin, Heidelberg, 2006.

[21] J.J. Mock, D.R. Smith, S. Schultz, Nano Lett. 3 (2003) 485-491.

[22] A.D. Mcfarland, M.A. Young, J.A. Dieringer, R.P. Van Duyne, J. Phys. Chem. B 109 (2005) 11279.

[23] E.M. Yeatman, Biosens. Bioelectron. 11 (1996) 635-649.

[24] E.M. Larsson, J. Alegret, M. Käll, D.S. Sutherland, Nano Lett. 7 (2007) 1256-1263.

[25] A.D. Mcfarland, M.A. Young, J.A. Dieringer, R.P. Van Duyne, Nano Lett. 3 (2003) 1057-1062.

[26] W.P. Chen, J.M. Chen, J. Opt. Soc. Am. A 71 (1981) 189.

[27] A.J. Haes, S. Zou, G.C. Schatz, R.P. Van Duyne, J. Phys. Chem. B 108 (2004) 109.

[28] A.J. Haes, S. Zou, G.C. Schatz, R.P. Van Duyne, J. Phys. Chem. B 108 (2004) 6961

[29] A.J. Haes, L. Chang, W.L. Klein, R.P. Van Duyne, J. Am. Chem. Soc. 127 (2005) 2264-2271.

[30] A.J. Haes, W.P. Hall, L. Chang, W.L. Klein, R.P. Van Duyne, Nano Lett. 4 (2004) 1029-1034.

[31] B.A. Chromy, R.J. Nowak, M.P. Lambert, K.L. Viola, L. Chang, P.T. Velasco, B.W. Jones, S.J. Fernandez, P.N. Lacor, P. Horowitz, C.E. Finch, G. A Krafft, W.L. Klein, Biochemistry 42 (2003) 12749.

[32] S. Yokogawa, S.P. Burgos, H.A. Atwater, Nano Lett. 12 (2012) 4349.

[33] A.D. Rakic, A.B. Djuristic, J.M. Elazar, M.L. Majewski, Appl. Opt. 37 (1998) 5271.

[34] T. Rindzevicius, Y. Alaverdyan, M. Kall, W.A. Murray, W.L. Barnes, J. Phys. Chem. C 111 (2007) 11806

[35] S. Szunerits, M.R. Das, R. Boukherroub, J. Phys. Chem. C 12 (2008) 8239-8243.

[36] A. Akjouj, G. Léveque, S. Szunerits, Y. Pennec, B. Djafari-Rouhani, R. Boukherroub, L. Dobrzynski, Surf. Sci. Rep. 68 (2013) 1-67.

[37] A.V. Whitney, J.W. Elam, S. Zou, A.V. Zinovev, P.C. Stair, G.C. Schatz, R.P. Van Duyne, J. Phys. Chem. B 109 (2005) 20522.

[38] O. Saison-Francioso, G. Leveque, R. Boukherroub, S. Szunerits, A. Akjouj, J. Phys. Chem. C 119 (2015) 28551-28559. 